\title{
EDITORIAL
}

\section{EPILEPSIA Y SALUD MENTAL: UN BINOMIO INDISOLUBLE}

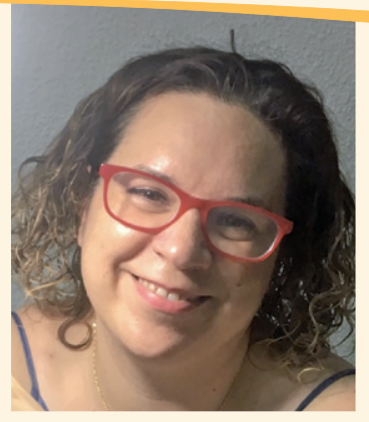

\section{MARÍA PALANCA CÁMARA}

Enfermera.

Unidad de Epilepsia

Refractaria.

Hospital Universitari i Politècnic La Fe. Valencia.
La International League Against Epilepsy (ILAE), en 2014, definió la epilepsia como una enfermedad cerebral caracterizada por la presencia de crisis epilépticas o un síndrome epiléptico, entendiendo por crisis epiléptica la aparición transitoria de signos y/o síntomas provocados por una actividad neuronal anómala excesiva o simultánea en el cerebro ${ }^{1}$.

Históricamente, la epilepsia fue tratada como algo sobrenatural, un castigo divino, o como un trastorno mental. Durante el siglo xIx, esta enfermedad era compartida entre ambas especialidades, la neurología y la psiquiatría. Con la llegada a mediados del siglo xx del desarrollo de la teoría electromagnética, la epilepsia se aceptó definitivamente como una enfermedad neurológica, pero con puentes de unión con la salud mental ${ }^{2}$ y, por ello, es necesario un abordaje multidisciplinario de esta enfermedad.

Existe una alta prevalencia de patología psiquiátrica en las personas con epilepsia, de entre el 20 y el $40 \% 3$.

La depresión es el trastorno más prevalente; lo sufren entre un 44 y un $63 \%$ de los enfermos de epilepsia. Además, se observa una tasa de suicidio de 5-25 veces mayor que para la población general. Respecto a la ansiedad, encontramos una prevalencia del 10-30\%, entre ansiedad generalizada y pánico, entre otros. Respecto a los trastornos de la personalidad, existe lo que conocemos como «personalidad epiléptica», en la que encontramos rasgos obsesivos, religiosidad marcada, irritabilidad, lentitud de procesamiento, etcétera ${ }^{3}$.

La psicosis en las personas con epilepsia es mayor que para la población general, teniendo una prevalencia del 8-10\%, con una duración muy variable, desde pocas horas hasta varias semanas ${ }^{3}$.

Todas estas alteraciones las podemos clasificar según la relación que guardan con las crisis epilépticas en: ictales, aquellas que aparecen durante la crisis epiléptica; periictales, como los signos y/o síntomas que observamos antes (las auras epilépticas) o después de la crisis epiléptica (delirio confusional); e interictales, que son las que no guardan una relación temporal directa con las crisis epilépticas ${ }^{4}$. 
Así, encontramos como trastornos preictales más habituales los síntomas o episodios depresivos, con un humor disfórico, o la psicosis. Los trastornos ictales son conocidos como auras psiquiátricas y, entre ellas, encontramos el miedo ictal, los síntomas ictales depresivos, o la psicosis con componente de alucinaciones o estados paranoides. Por último, los trastornos posictales más habituales suelen ser también los síntomas depresivos, así como ansiedad y los fenómenos psicóticos ${ }^{4}$.

No menos importantes son las crisis no epilépticas (CNEP), de origen psicógeno, conocidas como un cambio brusco en la conducta que recuerda a una crisis epiléptica, pero en las que no hay una causa orgánica, es decir, no aparecen los cambios en el electroencefalograma propios de una crisis epiléptica. En las unidades de epilepsia, encontramos que entre un 17 y un $30 \%$ de las personas diagnosticadas de epilepsia que no responden a fármacos antiepilépticos presentan CNEP de origen psicógeno. En su mayoría, son mujeres, con una edad de inicio entre los 20 y los 30 años, diagnosticadas de algún trastorno psiquiátrico hasta en un $70 \%$ de los casos, siendo los más frecuentes los trastornos del ánimo, los trastornos por abuso de sustancias, el estrés postraumático, la ansiedad y los trastornos disociativos, entre otros. Con todo esto, se ha visto como un espectro de enfermedades interconectadas compuestas por trastornos de somatización, conversión y episodios disociativos ${ }^{5}$.

Por todo ello, las enfermeras tanto de neurología como de salud mental, cuando cuidamos de personas con epilepsia, tal y como hemos visto, debemos tener en cuenta este binomio entre ambas especialidades para mejorar el cuidado de nuestros pacientes y prevenir complicaciones.

\section{BIBLIOGRAFÍA}

1. Fisher RS, Acevedo C, Arzimanoglou A, Bogacz A, Cross JH, Elger CE, et al. ILAE official report: a practical clinical definition of epilepsy. Epilepsia. 2014;55(4):475-82.

2. Reynolds EH, Trimble MR. Epilepsy, psychiatry, and neurology. Epilepsia. 2009;50 Suppl 3:50-5.

3. Burneo JG, Mayor LC, Quijano CA. Psiquiatría y epilepsia. Rev de Neuro-Psiquiat. 2005;68(1-2):83-92.

4. Rubio Granero T, Iranzo Tatay C, Livianos Aldana L. Psiquiatría y epilepsia. En: Carreno Martínez M (coord.). Tratado de epilepsia. Madrid: Luzán 5, S.A.; 2011. p. 257-72.

5. Baillès E, Pintor L, Torres X, Fernández-Egea E, De Pablo J, Arroyo S. Patología psiquiátrica en pacientes con crisis no epilépticas psicógenas derivados a una unidad de epilepsia en un hospital general. Actas Esp Psiquiatr. 2004;32(2): 76-81. 